\title{
Sequential Temporary and Permanent Control of Boolean Networks
}

\author{
Cui $\mathrm{Su}^{1}$ and Jun Pang ${ }^{1,2}$ \\ 1 Interdisciplinary Centre for Security, Reliability and Trust, University of \\ Luxembourg, Esch-sur-Alzette, Luxembourg \\ 2 Faculty of Science, Technology and Medicine, University of Luxembourg, \\ Esch-sur-Alzette, Luxembourg \\ firstname. lastname@uni.lu
}

\begin{abstract}
Direct cell reprogramming makes it feasible to reprogram abundant somatic cells into desired cells. It has great potential for regenerative medicine and tissue engineering. In this work, we study the control of biological networks, modelled as Boolean networks, to identify control paths driving the dynamics of the network from a source attractor (undesired cells) to the target attractor (desired cells). Instead of achieving the control in one step, we develop attractor-based sequential temporary and permanent control methods (AST and ASP) to identify a sequence of interventions that can alter the dynamics in a stepwise manner. To improve their feasibility, both AST and ASP only use biologically observable attractors as intermediates. They can find the shortest sequential control paths and guarantee $100 \%$ reachability of the target attractor. We apply the two methods to several real-life biological networks and compare their performance with the attractor-based sequential instantaneous control (ASI). The results demonstrate that AST and ASP have the ability to identify a richer set of control paths with fewer perturbations than ASI, which will greatly facilitate practical applications.
\end{abstract}

Keywords: Boolean networks $\cdot$ cell reprogramming $\cdot$ attractors $\cdot$ node perturbations $\cdot$ sequential control.

\section{Introduction}

Direct cell reprogramming, also called transdifferentiation, has provided a great opportunity for treating the most devastating diseases that are caused by a deficiency or defect of certain cells. It allows us to harness abundant somatic cells and transform them into desired cells to restore the structure and functions of damaged organs. However, the identification of efficacious intervention targets hinders the practical application of direct cell reprogramming.

Conventional experimental approaches are usually prohibited due to the high complexity of biological systems and the high cost of biological experiments [28]. Mathematical modelling of biological systems paves the way to study mechanisms of biological processes and identify therapeutic targets with formal reasoning and tools. Among various modelling frameworks, Boolean network (BN) 
has a distinct advantage $[6,7]$. It provides a qualitative description of biological systems and thus evades the parametrisation problem, which often occurs in quantitative modelling, such as networks of ordinary differential equations (ODEs). In BNs, molecular species (genes, transcription factors, etc.) are assigned binary-valued nodes, being either ' 0 ' or ' 1 '. The value of ' 0 ' describes the absence or inactive state of a species, whereas ' 1 ' represents the presence or active state. Activation/inhibition regulations between species are encoded as Boolean functions, which determine the evolution of the nodes. The dynamics of a BN evolves in discrete time under one of the updating schemes, such as synchronous or asynchronous updating schemes. The asynchronous updating scheme is considered more realistic than the synchronous one, since it non-deterministically updates one node at each time step and therefore can capture different biological processes at different time scales [19]. The long-run behaviour of the network dynamics is described as attractors, to one of which the network eventually settles down. Attractors are used to characterise cellular phenotypes or functional cellular states [5], such as proliferation, differentiation or apoptosis etc. In the context of BNs, direct cell reprogramming is equivalent to a source-target control problem: identifying a set of nodes, the perturbation of which can drive the network dynamics from a source attractor to the desired attractor.

The non-determinism of the asynchronous dynamics of BNs contributes to a better depiction of biological systems. As a result, it makes the control problem more challenging and renders the control methods designed for synchronous BNs inapplicable $[8,31,20]$. Another major obstacle to the control of BNs is the infamous state explosion problem - the state space is exponential in the size of the network. It prohibits the scalability and minimality of the control methods for asynchronous BNs $[30,10]$. The limitations of the existing methods motivate us to work on efficient and efficacious methods for the minimal source-target control of asynchronous BNs. There are different strategies to solve the control problem. Based on the control steps, we have one-step control and sequential control. One-step control applies all the perturbations simultaneously for one time, while sequential control identifies a sequence of perturbations that are applied at different time steps. In particular, we are interested in the sequential control that only adopts attractors as intermediates, called attractor-based sequential control. Rapid development of gene editing techniques enables us to silence or overexpress the expression of genes for different periods of time, thus, we have instantaneous, temporary and permanent perturbations. Instantaneous perturbations are applied instantaneously; temporary perturbations are applied for sufficient time steps and then released; permanent perturbations are applied for all the following steps. So far, we have developed methods for the minimal one-step instantaneous, temporary and permanent control (OI, OT and OP) [21, $22,26]$ and the attractor-based sequential instantaneous control (ASI) [11]. In this work, we focus on the attractor-based sequential temporary and permanent control (AST and ASP).

Due to the intrinsic diversity and complexity of biological systems, no single control method can perfectly suit all cases. Thus, it is of great importance to 
explore more strategies to provide a number of cautiously selected candidates for later clinical validations. AST and ASP integrate promising factors: attractorbased sequential control and temporary/permanent control. Attractor-based sequential control is more practical than the general sequential control [12], where any state can play the role of intermediate states. Moreover, temporary and permanent controls have proved their potential in reducing the number of perturbations [26]. In this work, we continue to develop efficient methods to solve the AST and ASP control problems. We apply our methods for AST and ASP to several biological networks to show their ability in finding new control paths with fewer perturbations compared to our previous methods $[21,22,26,11]$. We believe our new methods can provide a better understanding of the mechanismof-action of interventions and improve the efficiency of translating identified reprogramming paths into practical applications.

\section{Preliminaries}

In this section, we give preliminary notions of Boolean networks.

\section{$2.1 \quad$ Boolean networks}

A Boolean network (BN) describes elements of a dynamical system with binaryvalued nodes and interactions between elements with Boolean functions. It is formally defined as:

Definition 1 (Boolean networks). A Boolean network is a tuple $G=(X, F)$ where $X=\left\{x_{1}, x_{2}, \ldots, x_{n}\right\}$, such that $x_{i}, i \in\{1,2, \ldots, n\}$ is a Boolean variable and $F=\left\{f_{1}, f_{2}, \ldots, f_{n}\right\}$ is a set of Boolean functions over $X$.

For the rest of the exposition, we assume that an arbitrary but fixed network $G=(X, F)$ of $n$ variables is given to us. For all occurrences of $x_{i}$ and $f_{i}$, we assume $x_{i}$ and $f_{i}$ are elements of $X$ and $F$, respectively. A state $s$ of $G$ is an element in $\{0,1\}^{n}$. Let $S$ be the set of states of $G$. For any state $s=$ $(s[1], s[2], \ldots, s[n])$, and for every $i \in\{1,2, \ldots, n\}$, the value of $s[i]$, represents the value that the variable $x_{i}$ takes when the network is in state $s$. For some $i \in\{1,2, \ldots, n\}$, suppose $f_{i}$ depends on $x_{i_{1}}, x_{i_{2}}, \ldots, x_{i_{k}}$. Then $f_{i}(s)$ denotes the value $f_{i}\left(s\left[i_{1}\right], s\left[i_{2}\right], \ldots, s\left[i_{k}\right]\right)$. For two states $s, s^{\prime} \in S$, the Hamming distance between $s$ and $s^{\prime}$ is denoted as $\mathrm{hd}\left(s, s^{\prime}\right)$.

Definition 2 (Control). A control $C$ is a tuple $(\mathbb{D}, \mathbb{1})$, where $\mathbb{D}, \mathbb{1} \subseteq\{1,2, \ldots, n\}$ and $\mathbb{D}$ and $\mathbb{1}$ are mutually disjoint (possibly empty) sets of indices of nodes of a Boolean network $G$. The size of the control $C$ is defined as $|C|=|\mathbb{D}|+|\mathbb{1}|$. Given a state $s \in S$, the application of $C$ to $s$ is defined as a state $s^{\prime}=C(s)\left(s^{\prime} \in S\right)$, such that $s^{\prime}[i]=0=1-s[i]$ if $i \in \mathbb{O}$, and $s^{\prime}[i]=1=1-s[i]$ if $i \in \mathbb{1}$.

Definition 3 (Boolean networks under control). Let $C=(\mathbb{O}, \mathbb{1})$ be a control and $G=(X, F)$ be a Boolean network. The Boolean network $G$ under control $C$, denoted as $\left.G\right|_{C}$, is defined as a tuple $\left.G\right|_{C}=(\hat{X}, \hat{F})$, where $\hat{X}=$ 
$\left\{\hat{x}_{1}, \hat{x}_{2}, \ldots, \hat{x}_{n}\right\}$ and $\hat{F}=\left\{\hat{f}_{1}, \hat{f}_{2}, \ldots, \hat{f}_{n}\right\}$, such that for all $i \in\{1,2, \ldots, n\}$ :

(1) $\hat{x}_{i}=0$ if $i \in \mathbb{O}, \hat{x}_{i}=1$ if $i \in \mathbb{1}$, and $\hat{x}_{i}=x_{i}$ otherwise;

(2) $\hat{f}_{i}=0$ if $i \in \mathbb{O}, \hat{f}_{i}=1$ if $i \in \mathbb{1}$, and $\hat{f}_{i}=f_{i}$ otherwise.

The state space of $\left.G\right|_{C}$, denoted $\left.S\right|_{C}$, is derived by fixing the values of the variables in the set $C$ to their respective values and is defined as $\left.S\right|_{C}=\{s \in$ $S \mid s[i]=1$ if $i \in \mathbb{1}$ and $s[j]=0$ if $j \in \mathbb{O}\}$. Note that $\left.S\right|_{C} \subseteq S$. For any subset $S^{\prime}$ of $S$ we let $\left.S^{\prime}\right|_{C}=\left.S^{\prime} \cap S\right|_{C}$.

\subsection{Dynamics of Boolean networks}

In this section, we define several notions that can be interpreted on both $G$ and $\left.G\right|_{C}$. We use the generic notion $G=(X, F)$ to represent either $G=(X, F)$ or $\left.G\right|_{C}=(\hat{X}, \hat{F})$. A Boolean network $G=(X, F)$ evolves in discrete time steps from an initial state $s_{0}$. Its state changes in every time step according to the update functions $F$ and the update scheme. Different updating schemes lead to different dynamics of the network $[14,32]$. In this work, we are interested primarily in the asynchronous updating scheme - at each time step, one node is randomly selected to update its value based on its Boolean function. We define asynchronous dynamics formally as follows:

Definition 4 (Asynchronous dynamics of Boolean networks). Suppose $s_{0} \in S$ is an initial state of $G$. The asynchronous evolution of $G$ is a function $\xi: \mathbb{N} \rightarrow \wp(S)$ such that $\xi(0)=\left\{s_{0}\right\}$ and for every $j \geq 0$, if $s \in \xi(j)$ then $s^{\prime} \in \xi(j+1)$ is a possible next state of $s$ iff either $\mathrm{hd}\left(s, s^{\prime}\right)=1$ and there exists an $i$ such that $s^{\prime}[i]=f_{i}(s)=1-s[i]$ or $\mathrm{hd}\left(s, s^{\prime}\right)=0$ and there exists an $i$ such that $s^{\prime}[i]=f_{i}(s)=s[i]$.

It is worth noting that the asynchronous dynamics is non-deterministic and thus it can capture biological processes happening at different classes of time scales. Henceforth, when we talk about the dynamics of $G$, we shall mean the asynchronous dynamics as defined above. The dynamics of a Boolean network can be described as a transition system (TS).

Definition 5 (Transition system of Boolean networks). The transition system of a Boolean network $G$, denoted as $T S$, is a tuple $(S, E)$, where the vertices are the set of states $S$ and for any two states $s$ and $s^{\prime}$ there is a directed edge from $s$ to $s^{\prime}$, denoted $s \rightarrow s^{\prime}$ iff $s^{\prime}$ is a possible next state of $s$ according to the asynchronous evolution function $\xi$ of $G$.

A path $\sigma$ from a state $s$ to a state $s^{\prime}$ is a (possibly empty) sequence of transitions from $s$ to $s^{\prime}$. Thus, $\sigma=s_{0} \rightarrow s_{1} \rightarrow \ldots \rightarrow s_{k}$, where $s_{0}=s$ and $s_{k}=s^{\prime}$. A path from a state $s$ to a subset $S^{\prime}$ of $S$ is a path from $s$ to any state $s^{\prime} \in S^{\prime}$. For a state $s \in S$, reach $(s)$ denotes the set of states $S^{\prime}$ such that there is a path from $s$ to any $s^{\prime} \in S^{\prime}$ in $T S$ and can be defined as the fixpoint of the successor operation which is often denoted as post*. Thus, $\operatorname{reach}(s)=\operatorname{post}^{*}(s)$.

The long-run behaviour of the dynamics of a Boolean network is characterised as attractors, defined as follows. 


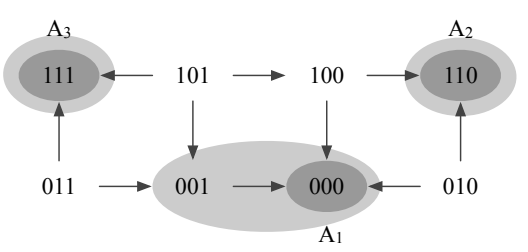

(a)

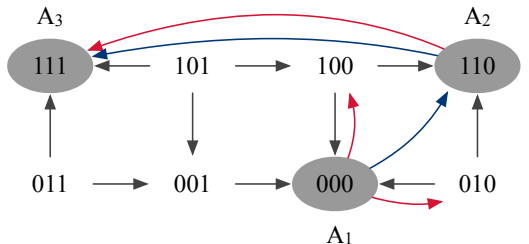

(b)

Fig. 1: (a) The transition system of the BN of Example 1; and (b) the control paths of Example 2 from attractor $A_{1}$ to attractor $A_{3}$. Paths indicated with blue (red) arrows are control paths with instantaneous (temporary/permanent) perturbations.

Definition 6 (Attractor). An attractor $A$ of TS is a minimal non-empty subset of states of $S$ such that for every $s \in A$, $\operatorname{reach}(s)=A$.

Any state which is not part of an attractor is a transient state. An attractor $A$ of $T S$ is said to be reachable from a state $s$ if $\operatorname{reach}(s) \cap A \neq \emptyset$. The network starting at any initial state $s_{0} \in S$ will eventually end up in one of the attractors of $T S$ and remain there forever unless perturbed. Thus, attractors are used to hypothesise cellular phenotypes or cell fates. We can easily observe that any attractor of $T S$ is a bottom strongly connected component of $T S$.

Let $\mathcal{A}$ be the set of attractors of TS. For an attractor $A \in \mathcal{A}$, we define the weak basin and the strong basin of $A$ to imply the commitment of states to $A$ in Definition 7. Intuitively, the weak basin of $A, \operatorname{bas}_{T S}^{W}(A)$, includes all the states $s$ from which there exists at least one path to $A$. It is possible that there also exist paths from $s$ to other attractor $A^{\prime}\left(A^{\prime} \neq A\right)$ of $T S$, while the notion of strong basin does not allow this. The strong basin of $A$, bas $_{T S}^{S}(A)$, consists of all the states from which there only exist paths to $A$.

Definition 7 (Weak basin and strong basin). The weak basin of $A$ is defined as bas ${ }_{T S}^{W}(A)=\{s \in S \mid \operatorname{reach}(s) \cap A \neq \emptyset\}$; and the strong basin of $A$ is defined as $\operatorname{bas}_{T S}^{S}(A)=\left\{s \in S \mid \operatorname{reach}(s) \cap A \neq \emptyset\right.$ and $\operatorname{reach}(s) \cap A^{\prime}=\emptyset$ for $\left.A^{\prime} \in \mathcal{A}, A^{\prime} \neq A\right\}$.

Example 1. Consider a network $G=(X, F)$, where $X=\left\{x_{1}, x_{2}, x_{3}\right\}, F=$ $\left\{f_{1}, f_{2}, f_{3}\right\}$, and $f_{1}=x_{2}, f_{2}=x_{1}$ and $f_{3}=x_{2} \wedge x_{3}$. Its transition system $T S$ is given in Fig. 1a. This network has three attractors that are marked with dark grey nodes, including $A_{1}=\{000\}, A_{2}=\{110\}$, and $A_{3}=\{111\}$. The strong basin of each attractor is marked as the light grey region. The weak basin of $A_{1}$ includes all the states except for states 110 and 111 . The weak basin of $A_{2}$ and $A_{3}$ are $\operatorname{bas}_{T S}^{W}\left(A_{2}\right)=\{010,100,101,110\}$ and $\operatorname{bas}_{T S}^{W}\left(A_{3}\right)=\{011,101,111\}$.

\section{Sequential Temporary and Permanent Control}

\subsection{The control problem}

As discussed in the introduction, direct cell reprogramming harnesses abundant somatic cells and reprograms them into desired cells. However, a major obstacle 


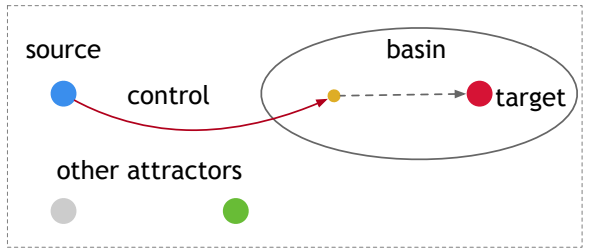

(a) One-step control

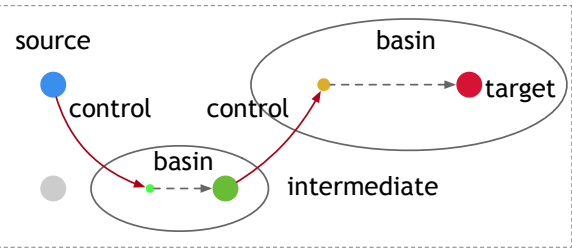

(b) Sequential control

Fig. 2: Two control strategies.

to the application of this novel technique lies in the identification of effective targets, the intervention of which can lead to desired changes. We aim to solve this problem by identifying key molecules based on BNs that model gene regulatory networks, such that the control of these molecules can drive the dynamics of a given network from a source attractor to the desired target attractor. We call it source-target control of BNs.

Thanks to the rapid advances in gene editing techniques, the control can be applied for different periods of time. Thus, we have instantaneous control, temporary control and permanent control. Let $A_{s}$ and $A_{t}$ denote the source and target attractors, respectively.

Definition 8 (Instantaneous, temporary and permanent controls).

(1) An instantaneous control is a control $C=(\mathbb{0}, \mathbb{1})$, such that by applying $C$ to a state $s \in A_{s}$ instantaneously, the network always reaches the target attractor $A_{t}$.

(2) A temporary control is a control $C=(\mathbb{O}, \mathbb{1})$, such that there exists a $t_{0} \geq 0$, for all $t \geq t_{0}$, the network always reaches the target attractor $A_{t}$ on the application of $C$ to a state $s \in A_{s}$ for $t$ steps.

(3) A permanent control is a control $C=(\mathbb{O}, \mathbb{1})$, such that the network always reaches the target attractor $A_{t}$ on the permanent application of $C$ to a state $s \in A_{s}$.

Temporary control applies perturbations for sufficient time and then is released, while permanent control maintains the perturbations for all the following time steps. Benefited from the extended intervention effects, temporary and permanent controls can potentially reduce the number of perturbations, which makes experiments easier to carry out and less costly [26].

The source-target control can also be achieved in one step or in multiple steps, called one-step control and sequential control, respectively. As illustrated in Fig. 2a, one-step control simultaneously applies all the required perturbations for one time (red arrow) to drive the network from a source state (blue node) to a state (yellow node), from which the network will converge to the target attractor in finite time steps (dashed line). In Fig. 2b, sequential control utilises other states as intermediates and identifies a sequence of perturbations, the application of which guides the network towards the target attractor in a stepwise manner. 
Considering difficulties in conducting clinical experiments, we are interested in attractor-based sequential control, where only biologically observable attractors can act as intermediates.

Given a source attractor $A_{s}$ and a target attractor $A_{t}$ of $T S$, the one-step control is formally defined as:

Definition 9 (One-step control). Compute a control $C_{A_{s} \rightarrow A_{t}}$, such that the application of $C_{A_{s} \rightarrow A_{t}}$ to a state $s \in A_{s}$ can drive the network towards $A_{t}$.

When the control $C_{A_{s} \rightarrow A_{t}}$ is the instantaneous, temporary or permanent control, we call it one-step instantaneous, temporary or permanent control (OI, OT or OP), respectively. To minimise the experimental costs, we are interested in the minimal solution $C_{A_{s} \rightarrow A_{t}}^{\min }$, which is the minimal such subset of $\{1,2, \ldots, n\}$. Let $\mathcal{A}$ be the attractors of $T S$. The attractor-based sequential control is defined as follows:

Definition 10 (Attractor-based sequential control). Find a sequence of attractors of TS, i.e. $\left\{A_{1}, A_{2}, \ldots, A_{m}\right\}$, where $A_{1}=A_{s}, A_{m}=A_{t}, A_{i} \neq A_{j}$ for any $i, j \in[1, m]$ and $2 \leq m \leq|\mathcal{A}|$, such that after the application of a sequence of minimal one-step controls $\left\{C_{A_{1} \rightarrow A_{2}}^{\mathrm{min}}, C_{A_{2} \rightarrow A_{3}}^{\min }, \ldots, C_{A_{m-1} \rightarrow A_{m}}^{\min }\right\}$, the network always eventually reaches $A_{m}$, i.e. $A_{t}$. We call it an attractor-based sequential control path, denoted as

$$
\begin{gathered}
\rho: A_{1} \stackrel{C_{A_{1} \rightarrow A_{2}}^{\min }}{\longrightarrow} A_{2} \stackrel{C_{A_{2} \rightarrow A_{3}}^{\min }}{\longrightarrow} A_{3} \stackrel{\cdots}{\longrightarrow} \ldots \stackrel{C_{A_{m-1} \rightarrow A_{m}}^{\min }}{\longrightarrow} A_{m} \\
\left(\left|C_{A_{1} \rightarrow A_{2}}^{\min }\right|+\left|C_{A_{2} \rightarrow A_{3}}^{\min }\right|+\ldots+\left|C_{A_{m-1} \rightarrow A_{m}}^{\min }\right|\right) \text { is the total number of perturbations. }
\end{gathered}
$$

Similarly, when the control $C_{A_{s} \rightarrow A_{t}}$ is the instantaneous, temporary or permanent control, we call it attractor-based sequential instantaneous, temporary or permanent control (ASI, AST or ASP), respectively.

We have developed efficient methods to tackle the minimal OI, OT and OP control $[21,22,26]$, as well as ASI control $[11,12]$. Considering the advantages of sequential control and temporary and permanent perturbations, in this paper, we shall develop methods to solve the AST and ASP control problems based on the methods for the minimal OT and OP control. Since the problems of the minimal OT and OP control are at least PSPACE-hard [26], the AST and ASP control are also computational difficult. We will demonstrate that based on efficient computation of the minimal OT and OP control, our methods for the AST and ASP control can also achieve a significant level of efficiency.

\subsection{Attractor-based sequential temporary control}

Algorithm 1 describes a procedure COMP_SEQ_TEMP to compute AST control paths within $k$ perturbations. This algorithm is based on our previously proposed methods, including the computation of weak basin and strong basin [22,21], denoted COMP_WEak_Basin and COMP_Strong_Basin, and the computation of the minimal OT control [26], denoted Comp_Temp_Control. Particularly, the procedure COMP_TEMP_CONTROL is based on Theorem 1. 


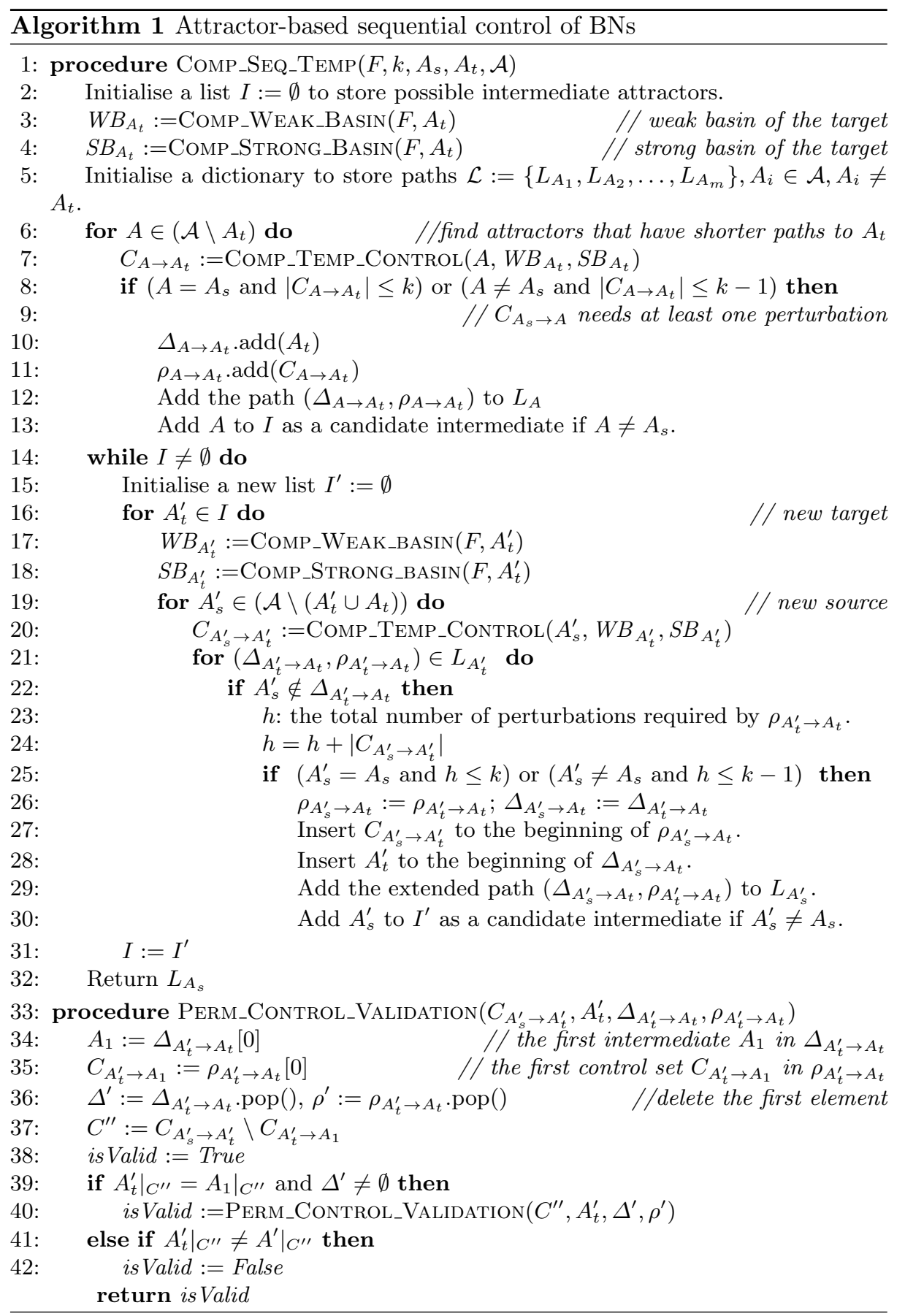


Theorem 1. A control $C=(\mathbb{D}, \mathbb{1})$ is a minimal temporary control from $s$ to $A_{t}$ iff (1) bas $\left.\underset{T S}{S}\left(A_{t}\right) \cap S\right|_{C} \neq \emptyset$ and $C(s) \in \operatorname{bas}_{\left.T S\right|_{C}}^{S}\left(\left.\operatorname{bas}_{T S}^{S}\left(A_{t}\right) \cap S\right|_{C}\right)$ and (2) $C$ is a minimal such subset of $\{1,2, \ldots, n\}$.

The procedure for the computation of AST control, COMP_SEQ_TEMP, takes as inputs the Boolean functions $F$, a threshold $k$ of the number of perturbations, a source attractor $A_{s}$, a target attractor $A_{t}$, and the set of attractors $\mathcal{A}$ of $T S$. It contains two parts.

The first part includes lines 2-13. For each attractor $A\left(A \in \mathcal{A}\right.$ and $\left.A \neq A_{t}\right)$, we generate a dictionary $L_{A}$ to save all the valid sequential control paths from $A$ to $A_{t}$ (line 5). We compute the minimal OT control set from $A$ to $A_{t}$, denoted $C_{A \rightarrow A_{t}} . C_{A \rightarrow A_{t}}$ is considered valid and saved to $L_{A}$ (line 8) if (1) $A$ is the source attractor $A_{s}$ and the number of perturbations $\left|C_{A \rightarrow A_{t}}\right|$ is not greater than $k$; or (2) $A$ is not $A_{s}$ and $\left|C_{A \rightarrow A_{t}}\right|$ is less or equal to $(k-1)$. If $A$ is an intermediate attractor $\left(A \neq A_{s}\right), C_{A_{s} \rightarrow A}$ requires at least one perturbation. Therefore, the size of $C_{A \rightarrow A_{t}}$ should not exceed $(k-1)$. $A$ is saved to $I$ as an intermediate attractor if $A \neq A_{s}$ and $\left|C_{A \rightarrow A_{t}}\right| \leq k-1$.

The second part includes lines 14-31. We extend the control paths computed in the previous part by iteratively taking every intermediate attractors $A_{t}^{\prime} \in I$ as a new target and computing the minimal temporary control from an attractor $A_{s}^{\prime}\left(A_{s}^{\prime} \in\left(\mathcal{A} \backslash\left(A_{t}^{\prime} \cup A_{t}\right)\right)\right)$ to $A_{t}^{\prime}$. Specifically, for each new target attractor $A_{t}^{\prime}$, we compute the minimal temporary control set $C_{A_{s}^{\prime} \rightarrow A_{t}^{\prime}}$ from $A_{s}^{\prime}$ to $A_{t}^{\prime}$ (line 20). Then, for every sequential path from $A_{t}^{\prime}$ to $A_{t}$, for instance $\left(\Delta_{A_{t}^{\prime} \rightarrow A_{t}}, \rho_{A_{t}^{\prime} \rightarrow A_{t}}\right)$, we verify whether $A_{s}^{\prime}$ can be appended to the beginning of $\Delta_{A_{t}^{\prime} \rightarrow A_{t}}$ to form a new path from $A_{s}^{\prime}$ to $A_{t}^{\prime}$ based on the following two conditions: (1) $A_{s}^{\prime}$ is not an intermediate attractor in the path $A_{t}^{\prime} \rightarrow \ldots \rightarrow A_{t}$ (line 22); and (2) the total number of perturbations of the new path $\Delta_{A_{s}^{\prime} \rightarrow A_{t}}$ should not exceed $k$ (or $k-1$ ) if $A_{s}^{\prime}=A_{s}$ (or $A_{s}^{\prime} \neq A_{s}$ ) (line 25). If both conditions are satisfied, we save the new path to $L_{A_{s}^{\prime}}$ (line 29) and add $A_{s}^{\prime}$ to $I^{\prime}$ as a new candidate intermediate if $A_{s}^{\prime} \neq A_{s}$ (line 30). After going through all the intermediate attractors in $I$ (lines $16-30$ ), we update the set of intermediate attractors $I$ and repeat steps at lines 14-31 until $I$ is an empty set.

\subsection{Attractor-based sequential permanent control}

In this section, we develop an algorithm to solve the ASP control problem. We have developed an algorithm to compute the minimal OP control [26], denoted as COMP_PERM_Control, based on Theorem 2.

Theorem 2. A control $C=(\mathbb{O}, \mathbb{1})$ is a minimal permanent control from $s$ to $A_{t}$ iff (1) $C(s) \in \operatorname{bas}_{\left.T S\right|_{C}}^{S}\left(A_{t}\right)$ and (2) $C$ is a minimal such subset of $\{1,2, \ldots, n\}$.

The procedure for ASP control explores in the same way as the procedure for AST control, COMP_SEQ_TEMP in Algorithm 1, to construct sequential paths, but it is more involved. It can be achieved by modifying procedure Comp_Seq_TEMP as follows. First, at lines 7 and 20, we simply replace the procedure COMP_TEMP_CONTROL with the procedure COMP_PERM_CONTROL. 
Second, when extending the sequential paths (lines $14-31$ ), besides the conditions at line 26, we add the procedure Perm_Control_Validation in Algorithm 1 to verify whether the control $C_{A_{s}^{\prime} \rightarrow A_{t}^{\prime}}$ can be inserted to the beginning of the path from $A_{t}^{\prime}$ to $A_{t}$. Because for each control step of AST, the temporary perturbations are released at one time point to retrieve the original transition system and let the network evolve spontaneously to the the intermediate/target attractor. But ASP adopts permanent control that will be maintained for all the following time steps. Therefore, when extending a permanent control $C$ to the beginning of a sequential path, it has to be verified whether the application of $C$ will affect the reachability of the following control steps. To avoid duplication, here we only give the explanations of the procedure PERM_CONTROL_VALIDATION. The purpose of this procedure is to verify whether the control $C_{A_{s}^{\prime} \rightarrow A_{t}^{\prime}}$ can be added to the beginning of $\rho_{A_{t}^{\prime} \rightarrow A_{t}}$ to form a new path $\rho_{A_{s}^{\prime} \rightarrow A_{t}}$ The verification is carried out recursively. Let us assume $\Delta_{A_{t}^{\prime} \rightarrow A_{t}}=\left\{A_{1}, A_{2}, \ldots, A_{t}\right\}$. The first intermediate attractor is $A_{1}$ and the control from $A_{t}^{\prime}$ to $A_{1}$ is $C_{A_{t}^{\prime} \rightarrow A_{1}}$. Since $C_{A_{s}^{\prime} \rightarrow A_{t}^{\prime}}$ and $C_{A_{t}^{\prime} \rightarrow A_{1}}$ may require to perturb the same node in the opposite way, we compute $\left(C_{A_{s}^{\prime} \rightarrow A_{t}^{\prime}} \backslash C_{A_{t}^{\prime} \rightarrow A_{1}}\right)$ and denote it as $C^{\prime \prime}$. If the projections of $A_{t}^{\prime}$ and $A_{1}$ to $C^{\prime \prime}$ are the same, $A_{1}$ is preserved under the permanent control $C^{\prime \prime}$ and we proceed to the remaining control steps (lines 39-40); otherwise, $C_{A_{s}^{\prime} \rightarrow A_{t}^{\prime}}$ is not a valid control (lines 41-42).

Example 2. To continue with Example 1, we compute the control paths from $A_{1}$ to $A_{3}$. As shown in Fig. 1b, the control path indicated with blue arrows, $A_{1} \stackrel{\left\{x_{1}, x_{2}\right\}}{\longrightarrow} A_{2} \stackrel{\left\{x_{3}\right\}}{\longrightarrow} A_{3}$, is the shortest ASI control, which requires three perturbations. AST and ASP have the same results indicated with red arrows in Fig. 1b: $A_{1} \underset{\left\{x_{1}\right\}}{\stackrel{\left\{x_{2}\right\}}{\longrightarrow}} A_{2} \stackrel{\left\{x_{3}\right\}}{\longrightarrow} A_{3}$, which require two perturbations in total.

\section{Evaluation}

In this section, we evaluate the performance of AST and ASP on several real-life biological networks. To demonstrate their efficacy, we compare their performance with ASI [11]. The minimal number of perturbations required by OI, OT and OP is set as the threshold $k$ of the number of perturbations for ASI, AST and ASP, respectively. In this way, the results will demonstrate whether AST and ASP can find sequential paths with fewer perturbations than ASI. All the methods are implemented as an extension of our software tool ASSA-PBN [14-16]. All the experiments are performed on a high-performance computing (HPC) platform, which contains CPUs of Intel Xeon Gold 6132 @2.6 GHz. We describe and discuss the results of the myeloid differentiation network [9] and the Th cell differentiation network [17] in detail (Sections 4.1 and 4.2), and we give an overview of the results of the other networks (Section 4.3).

\subsection{The myeloid differentiation network}

The myeloid differentiation network is constructed to model the differentiation process of common myeloid progenitors (CMPs) into four types of mature blood 


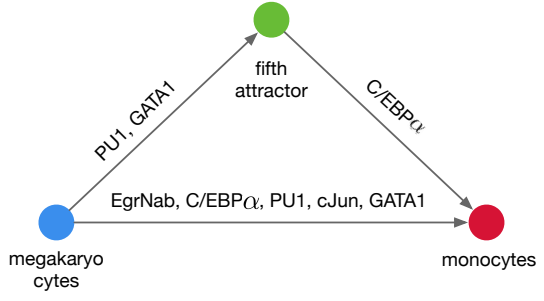

(a) ASI

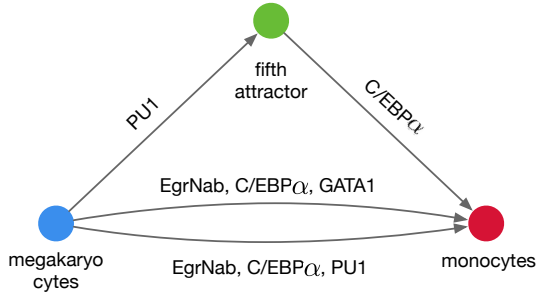

(b) AST/ASP

Fig. 3: Sequential control of the myeloid differentiation network.

cells [9]. With our attractor detection method [13], we identify six single-state attractors of the network, five of which are non-zero attractors (not all the nodes have a value of ' 0 '). It has been validated that expressions of four attractors correspond to microarray expression profiles of megakaryocytes, erythrocytes, granulocytes and monocytes [9]. The fifth attractor with the activation of PU1, cJun and EgrNab might be caused by pathological alterations [9] and the sixth attractor is an all-zero attractor, where all the nodes have a value of ' 0 '.

We take the conversion from megakaryocytes to monocytes as an example to show the performance of the methods. Note that the sixth attractor does not have a biological interpretation and mature erythrocytes in mammals do not have cell nucleus, therefore we do not consider these two attractors as intermediate attractors. Under this condition, the three methods (ASI, AST, ASP) identify both one-step and sequential paths as illustrated in Fig. 3. In particular, the results of AST and ASP are identical. We can see that the minimal OI control requires the activation of $\operatorname{EgrNab}, \mathrm{C} / \mathrm{EBP} \alpha, \mathrm{PU} 1$, cJun and the inhibition of GATA1 (Fig. 3a); while OT or OP can achieve the goal by either (1) the activation of EgrNab, C/EBP $\alpha$ and PU1; or (2) the activation of EgrNab and $\mathrm{C} / \mathrm{EBP} \alpha$, together with the inhibition of GATA1 (Fig. 3b). All the sequential paths need two steps, where the fifth attractor is adopted as an intermediate attractor. For the first step, ASI activates PU1 and inhibits GATA1, while AST or ASP only needs to activate PU1. When the network converges to the fifth attractor, all the three methods require to activate $\mathrm{C} / \mathrm{EBP} \alpha$. After that, the network will evolve spontaneously to the target attractor monocytes. Fig. 3 shows that AST and ASP are able to identify a path with only two perturbations, while ASI requires at least three perturbations.

The efficacy of the identified sequential temporary/permanent path is confirmed by the predictions in [9]. According to the expression profiles, both PU1 and $\mathrm{C} / \mathrm{EBP} \alpha$ are not expressed in MegE lineage (megakaryocytes and erythrocytes), while they are expressed in GM lineage (monocytes and granulocytes). In this network, no regulator can activate $\mathrm{C} / \mathrm{EBP} \alpha$ and $\mathrm{PU} 1$ is primarily activated by $\mathrm{C} / \mathrm{EBP} \alpha$. Therefore, $\mathrm{C} / \mathrm{EBP} \alpha$ has to be altered externally to reprogram MegE lineage to GM lineage. However, more perturbations are necessary to accurately reach the monocytes lineage. Sustained activation of PU1 and the 


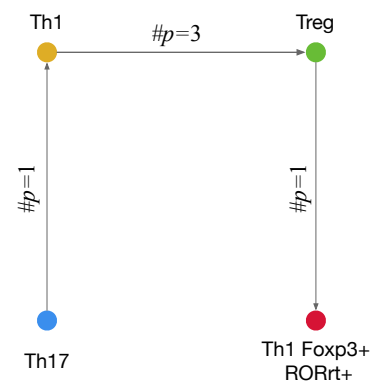

(a) ASI

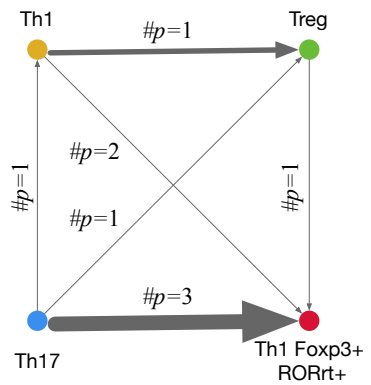

(b) AST

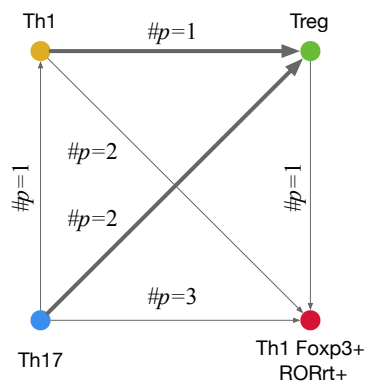

(c) ASP

Fig. 4: Sequential control of the Th cell differentiation network.

absence of $\mathrm{C} / \mathrm{EBP} \alpha$ guide the network to the fifth attractor, the expression of which differs with monocytes only in $\mathrm{C} / \mathrm{EBP} \alpha[9]$.

\subsection{The Th Cell differentiation network}

The T-helper (Th) cell differentiation network is a comprehensive model integrating regulatory network and signalling pathways that regulate Th cell differentiation [17]. This network consists of 12 single-state attractors under one initial condition and the attractors can be classified into different Th subtypes based on the expression of four master regulators [17].

Let Th17 and a Th1 subtype (Th1 Foxp3+ RORrt+) be the source and target attractors, respectively. For the purpose of illustration, we limit the number of control paths by only adopting Th1 and Treg as intermediate attractors. In addition, we set the node 'proliferation' as a non-perturbed node, since it denotes a cell fate and thus cannot be perturbed in reality. Fig. 4 describes the control paths identified by the three methods. The thickness of arrows implies the number of control sets and the equations $\# p=m$ above each arrow denotes the number of perturbations required by each step. All the methods identify sequential paths passing through Th1 and/or Treg. Fig. 4a only shows the shortest ASI path with five perturbations, while AST and ASP provide multiple paths with only two or three perturbations (Fig. 4b and Fig. 4c), demonstrating the advantages of AST and ASP in reducing the number of perturbations. Among the sequential paths of AST and ASP, only the AST path, Th17 $\stackrel{\text { IL27R }}{\longrightarrow}$ Treg $\stackrel{\text { TBET }}{\longrightarrow}$ the Th1 subtype, perturbs two nodes, all the other paths using either temporary or permanent perturbations need to perturb at least three nodes. This shows that AST has the potential to further reduce the number of perturbations compared to ASP. Moreover, in terms of the number of solutions, it is obvious that the arrows in Fig. 4b are thicker than those in Fig. 4c, which indicates that AST provides more solutions than ASP. 


\subsection{Other biological networks}

Besides the myeloid and Th cell differentiation networks, we also apply the three control methods to several other biological networks [4, 24, 18, 2, 23, 1, 3]. Here is a brief introduction of the networks.

- The cardiac gene regulatory network integrates key regulatory factors that play key roles in early cardiac development and FHF/SHF determination [4].

- The ERBB receptor-regulated G1/S transition network is built to identify efficacious targets for treating trastuzumab resistant breast cancer [24].

- The network of PC12 cell differentiation is built to capture the complex interplay of molecular factors in the decision of PC12 cell differentiation [18].

- The network of hematopoietic cell specification is constructed to capture the lymphoid and myeloid cell development [2].

- The network of bladder tumour is constructed to study mutually exclusivity and co-occurrence in genetic alterations [23].

- The pharmacodynamic model of bortezomib responses integrates major survival and apoptotic pathways in U266 cells to connect bortezomib exposure to multiple myeloma cellular proliferation [1].

- The network of a CD4 ${ }^{+}$immune effector $\mathrm{T}$ cell is constructed to capture cellular dynamics and molecular signalling under both immunocompromised and healthy settings [3].

Columns 2-4 of Table 1 summarise the number of nodes, edges and attractors contained in each network. For each network, we choose a pair of source and target attractors and compute control paths with ASI, AST and ASP.

Efficacy. For each pair of source and target attractors, all the control paths with at most $k$ perturbation are computed. For the purpose of comparison, in Table 1, columns 5-7 only summarise the minimal number of perturbations needed by each control method and columns 8-10 summarise the number of corresponding control paths. The results show that by extending the period of control time, AST and ASP have the ability to compute more control paths with fewer perturbations than ASI. This brings significant benefits for practical applications. First, fewer perturbations can reduce the experimental costs and make the experiments easier to conduct. Second, a richer set of control paths provides biologists more options to tackle diverse biological systems.

To further compare AST and ASP, AST is more appealing than ASP. As discussed in the previous subsection, the control of Th cell differentiation network (T-diff in Table 1) shows that AST has the potential to identify smaller control sets than ASP. For the other cases listed in Table 1, although AST requires the same number of perturbations as ASP, AST identifies more solutions than ASP. Apart from that, AST has an intrinsic advantage compared to ASP - temporary control will eventually be released and therefore can eliminate risks of unforeseen consequences, which may be caused by the permanent shift of the dynamics.

Efficiency. The last three columns of Table 1 give the computation time of ASI, AST and ASP. Although AST and ASP take longer time than ASI, they are still 


\begin{tabular}{|c|c|c|c|c|c|c|c|c|c|c|c|c|}
\hline \multirow{2}{*}{ network } & \multirow{2}{*}{$|V|$} & \multirow{2}{*}{$|E|$} & \multirow{2}{*}{$|\mathcal{A}|$} & \multirow{2}{*}{\multicolumn{3}{|c|}{ \#perturbations }} & \multicolumn{3}{|c|}{ \# paths } & \multicolumn{3}{|c|}{ time (seconds) } \\
\hline & & & & ASI & & & ASI & $\mathrm{SST}$ & $\mathrm{ASP}$ & ASI & AST & ASP \\
\hline lye & 11 & 30 & 6 & 3 & 2 & 2 & 1 & 1 & 1 & 0.006 & 0.034 & 0.038 \\
\hline & & 39 & 6 & 3 & 2 & 2 & & 3 & & & 0.6 & 0.65 \\
\hline $\mathrm{R}$ & 20 & 52 & 3 & 8 & 3 & 3 & 2 & 3 & & 0.007 & 0.249 & 0.319 \\
\hline $\mathrm{C}$ & $3:$ & 62 & 7 & 8 & 2 & 2 & 3 & 50 & 30 & 0.0 & 1. & $1.46^{\circ}$ \\
\hline $\mathrm{ISC}$ & 33 & 88 & 5 & 12 & 2 & 2 & 2 & 12 & 6 & 0.406 & 12.217 & 8.879 \\
\hline blade & 35 & 116 & 4 & 5 & 2 & 2 & 2 & 2 & & 0.139 & 0.709 & 0.676 \\
\hline bort & 67 & 1: & 5 & 3 & 2 & 2 & 1 & 4 & 2 & 1.900 & 105.184 & 119.138 \\
\hline T-di & 68 & 175 & 12 & 5 & 2 & 3 & 4 & 1 & 14 & 9.713 & 95.211 & 71.044 \\
\hline $\mathrm{CD} 4^{+}$ & 188 & 380 & 6 & 3 & 2 & 2 & 3 & 48 & 6 & 256.492 & 539.868 & 1304.490 \\
\hline
\end{tabular}

Table 1: Sequential control of several biological networks.

quite efficient and are capable of handling large-scale and comprehensive networks. In general, the computational time of the methods depends on the size of the network, the threshold of the number of perturbations $k$ and the number of existing solutions within the threshold. By increasing the threshold $k$, our methods can identify more candidate solutions at the cost of longer computational time. Currently, due to the lack of large and well-behaved networks, we are not yet able to find out the precise limit of our methods on the size of networks.

\section{Discussion}

We have demonstrated the potential strengths of AST and ASP, however, they are not warranted to be the best methods for all kinds of biological systems. Indeed, there is no control method that can perfectly solve all the control problems due to the intrinsic diversity and complexity of biological systems. Given a specific task, it is thus recommended to compute all the control paths with available control methods. Various sets of identified therapeutic targets serve as candidates, such that biologists can choose appropriate targets, the modulation of which will not disrupt physiological functions of biological systems.

Although the dynamics of asynchronous BNs are non-deterministic, our methods guarantee to find the shortest control paths with $100 \%$ reachability in silico. Experimental validation is necessary to verify their therapeutic efficacy in vivo. It is worth noticing that the consistency of the efficacy in silico and in vivo highly relies on the quality of the constructed BNs. The identified perturbations can effectively modulate the dynamics as expected, provided that the adopted network well captures the structural and dynamical properties of the real-life biological system. However, mathematical modelling of vastly complex biological systems is already a challenging task by itself in systems biology. We have spotted some flaws of the constructed networks in the literature during analysis, summarised as follows.

First, simulation is often used to evaluate the stable behaviour of dynamics in most of the works. However, simulation can hardly cover the entire transition 
system of a BN, which is exponential in the size of the network. As a consequence, the information on attractors is usually incomplete, especially for networks of medium or large sizes. This problem can be solved by using our attractor detection method $[13,29]$ to identify all the exact attractors of a network.

Second, we noticed that the attractors of some large networks are purely induced by input nodes. For instance, given a network with 2 input nodes (nodes without upstream regulators), it has $2^{2}$ attractors. Each attractor corresponds to one combination of the input nodes $(00,01,10,11)$. For such networks, the input nodes, that have different values in the source and target attractors, are the key nodes for modulating the dynamics. Such kind of networks may capture some activation or inhibition regulations, but they fail to depict the intrinsic mechanisms of biological processes.

Third, in some networks, cell phenotypes or cell fates, such as apoptosis, proliferation, and differentiation, are represented as marker nodes. Benefited from this, attractors can be classified based on the expressions of those nodes. A problem that often occurs is that there does not exist any control sets without perturbing these marker nodes, however, these nodes can not be perturbed in reality. Again, we hypothesise that these constructed networks do not reflect the intrinsic properties of biological systems.

Our methods $[13,29,22,26,11]$ can provide accurate information of the networks, such as the number and size of the attractors and potential sets of control nodes. Such information related to the network dynamics should be taken into account when inferring the networks by updating the Boolean functions or adding/deleting regulators.

\section{Conclusion and Future Work}

In this work, we have developed the AST and ASP control methods to identify sequential control paths for modulating the dynamics of biological systems. To make it practical, only biologically observable attractors are served as intermediates. We compared the performance of the two methods with ASI on a variety of biological networks. The results show that our new methods have apparent advantages in reducing the number of perturbations and enriching the diversity of solutions. Among the three sequential control methods (ASI, AST and ASP), AST is more preferable because it requires the fewest number of perturbations and it adopts temporary perturbations which will eventually be released and thus can evade unforeseen consequences that might be caused by permanent perturbations.

Until now, we have developed source-target control methods to alter the dynamics of BNs in different ways. Currently, we are working on a target control method to identify a subset of nodes, the intervention of which can transform any somatic cells to the desired cell. We also plan to study the control of probabilistic Boolean networks [25,27] based on our control methods for BNs. We believe our works can provide deep insights into regulatory mechanisms of biological processes and facilitate direct cell reprogramming. 
Acknowledgements. This work was partially supported by the project SECPBN funded by University of Luxembourg and the ANR-FNR project AlgoReCell (INTER/ANR/15/11191283).

\section{References}

1. Chudasama, V., Ovacik, M., Abernethy, D., Mager, D.: Logic-based and cellular pharmacodynamic modeling of bortezomib responses in u266 human myeloma cells. Journal of Pharmacology and Experimental Therapeutics 354(3), 448-458 (2015)

2. Collombet, S., van Oevelen, C., Ortega, J., Abou-Jaoudé, W., Di Stefano, B., Thomas-Chollier, M., Graf, T., Thieffry, D.: Logical modeling of lymphoid and myeloid cell specification and transdifferentiation. Proceedings of the National Academy of Sciences 114(23), 5792-5799 (2017)

3. Conroy, B.D., Herek, T.A., Shew, T.D., Latner, M., Larson, J.J., Allen, L., Davis, P.H., Helikar, T., Cutucache, C.E.: Design, assessment, and in vivo evaluation of a computational model illustrating the role of CAV1 in CD4+ T-lymphocytes. Frontiers in Immunology 5, 599 (2014)

4. Herrmann, F., Groß, A., Zhou, D., Kestler, H.A., Kühl, M.: A Boolean model of the cardiac gene regulatory network determining first and second heart field identity. PLOS ONE 7, 1-10 (10 2012)

5. Huang, S.: Genomics, complexity and drug discovery: insights from Boolean network models of cellular regulation. Pharmacogenomics 2(3), 203-222 (2001)

6. Kauffman, S.A.: Homeostasis and differentiation in random genetic control networks. Nature 224, 177-178 (1969)

7. Kauffman, S.A.: Metabolic stability and epigenesis in randomly constructed genetic nets. Journal of Theoretical Biology 22(3), 437-467 (1969)

8. Kim, J., Park, S., Cho, K.: Discovery of a kernel for controlling biomolecular regulatory networks. Scientific Reports 3(2223) (2013)

9. Krumsiek, J., Marr, C., Schroeder, T., Theis, F.J.: Hierarchical differentiation of myeloid progenitors is encoded in the transcription factor network. PLOS ONE 6(8), e22649 (2011)

10. Mandon, H., Haar, S., Paulevé, L.: Relationship between the reprogramming determinants of Boolean networks and their interaction graph. In: Proc. 5th Workshop on Hybrid Systems Biology. LNCS, vol. 9957, pp. 113-127. Springer (2016)

11. Mandon, H., Su, C., Haar, S., Pang, J., Paulevé, L.: Sequential reprogramming of Boolean networks made practical. In: Proc. 17th International Conference on Computational Methods in Systems Biology. LNCS, vol. 11773, pp. 3-19. SpringerVerlag (2019)

12. Mandon, H., Su, C., Pang, J., Paul, S., Haar, S., Paulevé, L.: Algorithms for the sequential reprogramming of Boolean networks. IEEE/ACM Transactions on Computational Biology and Bioinformatics 16(5), 1610-1619 (2019)

13. Mizera, A., Pang, J., Qu, H., Yuan, Q.: Taming asynchrony for attractor detection in large Boolean networks. IEEE/ACM Transactions on Computational Biology and Bioinformatics 16(1), 31-42 (2019)

14. Mizera, A., Pang, J., Su, C., Yuan, Q.: ASSA-PBN: A toolbox for probabilistic Boolean networks. IEEE/ACM Transactions on Computational Biology and Bioinformatics 15(4), 1203-1216 (2018) 
15. Mizera, A., Pang, J., Yuan, Q.: ASSA-PBN: a tool for approximate steady-state analysis of large probabilistic Boolean networks. In: Proc. 13th International Symposium on Automated Technology for Verification and Analysis. LNCS, vol. 9364, pp. 214-220. Springer (2015)

16. Mizera, A., Pang, J., Yuan, Q.: ASSA-PBN 2.0: A software tool for probabilistic Boolean networks. In: Proc. 14th International Conference on Computational Methods in Systems Biology. LNCS, vol. 9859, pp. 309-315. Springer (2016)

17. Naldi, A., Carneiro, J., Chaouiya, C., Thieffry, D.: Diversity and plasticity of th cell types predicted from regulatory network modelling. PLoS Computational Biology 6(9) (2010)

18. Offermann, B., Knauer, S., Singh, A., Fernández-Cachón, M.L., Klose, M., Kowar, S., Busch, H., Boerries, M.: Boolean modeling reveals the necessity of transcriptional regulation for bistability in PC12 cell differentiation. Frontiers in Genetics 7, 44 (2016)

19. Papin, J.A., Hunter, T., Palsson, B.O., Subramaniam, S.: Reconstruction of cellular signalling networks and analysis of their properties. Nature Reviews Molecular Cell Biology 6(2), 99 (2005)

20. Pardo, J., Ivanov, S., Delaplace, F.: Sequential reprogramming of biological network fate. In: Proc. 17th International Conference on Computational Methods in Systems Biology. pp. 20-41. Springer-Verlag (2019)

21. Paul, S., Su, C., Pang, J., Mizera, A.: A decomposition-based approach towards the control of Boolean networks. In: Proc. 9th ACM Conference on Bioinformatics, Computational Biology, and Health Informatics. pp. 11-20. ACM Press (2018)

22. Paul, S., Su, C., Pang, J., Mizera, A.: An efficient approach towards the sourcetarget control of Boolean networks. IEEE/ACM Transactions on Computational Biology and Bioinformatics (2020), accepted

23. Remy, E., Rebouissou, S., Chaouiya, C., Zinovyev, A., Radvanyi, F., Calzone, L.: A modeling approach to explain mutually exclusive and co-occurring genetic alterations in bladder tumorigenesis. Cancer Research 75(19), 4042-4052 (2015)

24. Sahin, Ö., Fröhlich, H., Löbke, C., Korf, U., Burmester, S., Majety, M., Mattern, J., Schupp, I., Chaouiya, C., Thieffry, D., et al.: Modeling ERBB receptor-regulated $\mathrm{G} 1 / \mathrm{S}$ transition to find novel targets for de novo trastuzumab resistance. BMC Systems Biology 3(1), 1 (2009)

25. Shmulevich, I., Dougherty, E.R.: Probabilistic Boolean Networks: The Modeling and Control of Gene Regulatory Networks. SIAM Press (2010)

26. Su, C., Paul, S., Pang, J.: Controlling large Boolean networks with temporary and permanent perturbations. In: Proc. 23rd International Symposium on Formal Methods. LNCS, vol. 11800, pp. 707-724. Springer-Verlag (2019)

27. Trairatphisan, P., Mizera, A., Pang, J., Tantar, A.A., Schneider, J., Sauter, T.: Recent development and biomedical applications of probabilistic Boolean networks. Cell Communication and Signaling 11, 46 (2013)

28. Wang, L.Z., Su, R.Q., Huang, Z.G., Wang, X., Wang, W.X., Grebogi, C., Lai, Y.C.: A geometrical approach to control and controllability of nonlinear dynamical networks. Nature Communications 7, 11323 (2016)

29. Yuan, Q., Mizera, A., Pang, J., Qu, H.: A new decomposition-based method for detecting attractors in synchronous Boolean networks. Science of Computer Programming 180, 18-35 (2019)

30. Zañudo, J.G.T., Albert, R.: Cell fate reprogramming by control of intracellular network dynamics. PLoS Computational Biology 11(4), e1004193 (2015) 
31. Zhao, Y., Kim, J., Filippone, M.: Aggregation algorithm towards large-scale Boolean network analysis. IEEE Transactions on Automatic Control 58(8), 19761985 (2013)

32. Zhu, P., Han, J.: Asynchronous stochastic Boolean networks as gene network models. Journal of Computational Biology 21(10), 771-783 (2014) 